\title{
Localization judgments under various levels of attention
}

\author{
YEHOSHUA TSAL and TAL BAREKET \\ Tel Aviv University, Tel Aviv, Israel
}

\begin{abstract}
We investigated the effects of attention on localization with two converging manipulations of attention. The results indicated that whereas attention improved localization, minimally attended stimuli were nonetheless localized fairly accurately. The distributions of localization responses around peripheral stimuli were asymmetric, with a greater dispersion along the axis linking fixation to stimulus location relative to its perpendicular axis. We propose that the unattended field comprises attentional receptive fields that are mediated by coarse location detectors. We speculate that attention produces fine localization by facilitating computations that integrate the relative activation of overlapping detectors.
\end{abstract}

Questions concerning the effects of attention on detection and identification of specific stimulus aspectssuch as shape, color, or orientation - have been extensively investigated in the literature, and it is widely accepted that attention improves performance on these tasks. In contrast, few studies have assessed the effects of attention on localization. This relative lack of experimental interest does not reflect a consensus on the issue. Indeed, whereas some investigators posit that no localization whatsoever is possible without attention, others assume that localization does not require attention. On the one hand, Treisman's FIT (e.g., Treisman \& Gormican, 1988), for instance, assumes that without attention features are free floating; that is, they are registered without location information. With attention, locational certainty depends on the breadth of the locational focus: The smaller the area on which attention is focused, the more accurately its features can be localized and conjoined.

On the other hand, the ubiquitous use of exogenous cues to direct attention (e.g., Jonides, 1981) implies that stimuli can be localized preattentively. If a cue can attract attention automatically to its location, one must assume that the cue is localized before attention "arrives" at its location. Furthermore, some studies suggest that accurate, preattentive, location-based judgments can take place without attention (e.g., Sagi \& Julesz, 1985).

A third, intermediate, view suggests that coarse localization is possible without attention, whereas fine localization requires attention (Cohen \& Ivry, 1989, 1991; Newby \& Rock, 2001; Tsal, Meiran, \& Lamy, 1995; Tsal $\&$ Shalev, 1996). This view has received indirect support by showing that conjunctive search slopes reflected preat-

This research was supported by Grant 0610710551 from the Israel Academy of Sciences. We are grateful to Dominique Lamy, Yonatan Goshen, and three anonymous reviewers for their helpful comments on an earlier draft. Correspondence should be addressed to Y. Tsal, Department of Psychology, Tel Aviv University, Ramat Aviv, Tel Aviv 69978 Israel (e-mail: jehoshua@freud.tau.ac.il). tentive processing as long as the items were separated by more than $0.78^{\circ}$ of visual angle (Cohen \& Ivry 1989). Similarly, illusory conjunctions between unattended items occurred only if the items were separated by less than $1^{\circ}$ of visual angle (Cohen \& Ivry, 1991).

The first attempt to determine the specific effects of attention on location perception using a direct measure of localization was reported by Tsal and Meiran (1993). In this study, participants localized a briefly presented letter and attention was manipulated by precuing one of three regions on the screen most likely to contain the target. The spread of localization responses was significantly smaller on valid- than on invalid-cue trials, indicating that attention improved location perception. In a later study, Tsal and Bareket (1999) replicated these results and, using additional measures, revealed that responses displayed significantly greater dispersion along the horizontal axis than along the vertical axis. However, since the stimuli were presented along the horizontal axis, it is not clear whether this result is unique to this axis or represents a more general characteristic of localization distributions in the periphery of the visual field. Prinzmetal, Amiri, Allen, and Edwards (1998) used the same method to assess the effects of attention on localization but employed the dual-task technique to manipulate attention. They showed reduction in localization accuracy when less attention was allocated to the localization task.

The results of the above three studies have generally been interpreted as showing that attention improves location perception, thus suggesting that attention may be a prerequisite for stimulus localization. Indeed, the finding that attention improves a given perceptual operation has often been used in the literature to conclude that this operation could not be performed preattentively (e.g., in Treisman's FIT, the finding that feature integration is influenced by attention is taken to mean that no feature integration could be performed preattentively). However, it should be emphasized (e.g., Tsal, 1989) that the ques- 
tion concerning preattentive perception and that concerning the effects of attention on perception should be treated orthogonally.

Indeed, a close examination of the above three localization studies' data indicates that irrespective of the significant effects of attention on localization, stimuli were localized fairly accurately even under the low-attention conditions, suggesting that coarse localization is possible with minimal attention. Unfortunately, however, these studies could not provide unequivocal support for this view since none ensured absolute location uncertainty under the low-attention conditions. The cost-benefit technique used in the studies of Tsal and Meiran (1993) and Tsal and Bareket (1999) could not ensure that on invalid trials the stimulus was maximally unattended and, furthermore, the large circle enclosing the target could facilitate localization. Thus, the limited spread of localization responses on invalid trials could reflect the benefit of some attentional resources or the facilitating information regarding the relative position of the target within the enclosing circle. In the study of Prinzmetal et al. (1998), all target dots were presented at the same distance from fixation, thus eliminating all spatial uncertainty along the tangential axis. Since no analysis was conducted to separate the radial from the tangential dispersion, it is not clear whether the results reflect good localization with minimal attention or the facilitation of partial location certainty with respect to stimulus location. Furthermore, in none of the above studies was attention maximally focused on the target in the highattention conditions. In the studies of Tsal and Meiran (1993) and Tsal and Bareket (1999), some attentional resources were likely to be directed to the noncued circles, which had a $30 \%$ probability of containing the target. Furthermore, the large cued circle provided only general, rather than specific, location information. In the study of Prinzmetal et al. (1998), the high-attention condition was essentially a distributed- rather than a focused-attention condition since the target could appear at any peripheral location without a preceding cue. Including a strong manipulation of focused attention may be important in order to establish a baseline scale against which the localization performance with minimal attention could be compared.

In the present study, we used two converging operations to assess the effects of attention on localization performance. In the first experiment, we employed a cuing method that was superior to the one used by Tsal and Meiran (1993) and Tsal and Bareket (1999) in that we used a $100 \%$-valid cue to maximize attention in the focusedattention condition and no informative cue to minimize attention in the distributed-attention condition. In the second experiment, we used the dual-task technique to investigate whether limiting attentional resources would reduce localization precision. Participants performed a difficult localization task either alone or in conjunction with a highly demanding task. Unlike in the study of Prinzmetal et al. (1998), however, we maximized location uncertainty by presenting the target in a random position within one of four diagonal regions, the diameter of each subtending $4^{\circ}$ of visual angle. This advantage is especially important here since we wanted to obtain a faithful quantitative estimate of the spread of localization responses for minimally attended stimuli with maximal location uncertainty.

\section{EXPERIMENT 1}

In the first experiment, participants were required to localize and identify a target that appeared in one of five possible locations. In the focused-attention condition, a single precue directed attention to the designated stimulus location. In the distributed-attention condition, all five locations were precued. In the focused-attention condition, we used a $100 \%$-valid abrupt onset, since our purpose, of course, was not to examine the separate effects of endogenous and exogenous cuing, but rather to combine the two in order to maximize attentional resources at the cued target location.

\section{Method}

Participants. The participants were 16 undergraduates from Tel Aviv University who participated to fulfill a course requirement. All had normal or corrected-to-normal vision.

Stimuli and Procedure. The stimulus presentation and data collection were controlled by an IBM PC/AT with a VGA graphic display. A chinrest was used to stabilize viewing distance at $50 \mathrm{~cm}$, so that $1 \mathrm{~cm}$ on the display corresponded to $1.15^{\circ}$ of visual angle. On each trial, the participants were required to localize and identify a single brown letter that was randomly selected from the following set: A, E, Q, W, and Z. Each letter subtended a visual angle of $0.39^{\circ}$ in height and $0.26^{\circ}$ in width. The letter appeared equally frequently at each of five general locations, around the center or around each corner of an imaginary square, the side of which subtended $7.8^{\circ}$ of visual angle. In each of the five locations, the letter appeared in a random position within an area, the diameter of which subtended $4^{\circ}$ of visual angle. The participants were tested under two conditions. In the focused-attention condition, the location of the letter was precued, whereas in the distributed-attention condition, all five locations were precued. The cue was a small open circle subtending $0.2^{\circ}$ of visual angle. Each trial consisted of the following sequence of events. First, a fixation cross was presented for $1.5 \mathrm{sec}$. Then, the cue(s) appeared for $50 \mathrm{msec}$. Then, following either a long interstimulus interval (ISI; $70 \mathrm{msec})$ or a short ISI $(20 \mathrm{msec})$, the target was presented for $50 \mathrm{msec}$. The participants first localized the letter by touching the screen with their preferred hand with a thin marker. The cursor was then moved by the experimenter to the perceived stimulus location, and the response was recorded. Then, the participants identified the letter by pressing the corresponding key on the extended keyboard. Auditory signals were used to provide feedback for correct identification and for good localization (a deviation smaller than $0.6^{\circ}$ from the stimulus position) on every trial. Each block was preceded by 30 practice trials, after which four 60 -trial blocks were presented, corresponding to the factorial combination of the two attention conditions and two ISIs. The order of block presentation was randomized across participants.

\section{Results and Discussion}

Initial inspections of the data suggested no differential dispersion along the horizontal and vertical axes but rather a substantially greater localization dispersion along the diagonal axis linking fixation to stimulus location (the radial axis) than the axis perpendicular to it (the tangential axis). Statistical analyses indeed confirmed this 
observation for the focused-attention condition $[F(1,15)=$ $4.32, p=.0551]$ and the distributed-attention condition $[F(1,15)=11.59, p<.005]$. This finding indicates that the elongated distribution of localization responses is not unique to the horizontal dimension, but instead characterizes the differential spread along the radial and tangential axes of peripheral stimuli in general. Thus, in addition to the overall analyses, the localization dispersions around peripheral stimuli were computed separately along the two diagonal axes. Table 1 shows the mean localization deviations of central and peripheral stimuli for the two ISIs. The overall analysis of variance (ANOVA) of eccentricity (central vs. peripheral) $\times$ attention (focused vs. distributed) $\times$ ISI (short vs. long) $\times$ participants indicated that central stimuli were localized more accurately than peripheral ones $[F(1,15)=40.36, p<.001]$ and stimuli in the focused-attention condition were localized more accurately than those in the distributed-attention condition $[F(1,15)=11.60, p<.004]$. The effect of ISI was not significant, and none of the interactions reached statistical significance. Separate ANOVAs performed on localization dispersions along the two axes revealed that attention reduced the dispersion along the radial axis $[F(1,15)=6.75, p<.02]$, but not along the tangential axis. Table 2 presents the mean proportions of correct letter identifications under the various conditions. An overall analysis assessing the various effects on identification showed that all main effects were significant. Letters were identified more accurately in the long ISI presentation than in the short ISI presentation $[F(1,15)=$ $6.75, p<.02]$. Central letters were identified more accurately than peripheral ones $[F(1,15)=256.6, p<$ $.001]$, and letters were identified more accurately in the focused-attention condition than in the distributed-attention condition $[F(1,15)=7.00, p<.02]$. None of the interactions reached significance.

The present findings support the notion that coarse localization is possible with minimal attention. The results show a small mean dispersion of localization responses for the distributed-attention condition $\left(0.50^{\circ}\right.$, center; $0.64^{\circ}$, periphery). Moreover, this dispersion is only slightly larger than the one for the focused-attention condition $\left(0.44^{\circ}\right.$, center; $0.60^{\circ}$, periphery), which highly maximized attentional resources at the cued location. Hence,

Table 1

Experiment 1: Mean Localization Deviations in Degrees of Visual Angle

\begin{tabular}{cccccc}
\hline & \multicolumn{2}{c}{ Attended } & & \multicolumn{2}{c}{ Unattended } \\
\cline { 2 - 3 } \cline { 5 - 6 } & $M$ & $S D$ & & $S D$ \\
\hline Short ISI & & & & \\
Central & .45 & .19 & .53 & .24 \\
Peripheral & .61 & .15 & .63 & .12 \\
Long ISI & & & & \\
Central & .43 & .17 & .47 & .19 \\
$\quad$ Peripheral & .59 & .12 & .65 & .15 \\
\hline
\end{tabular}

Note-ISI, interstimulus interval.
Table 2

Experiment 1: Mean Proportions

of Correct Letter Identifications

\begin{tabular}{cccccc}
\hline \multicolumn{3}{c}{ of Correct Letter Identifications } \\
\cline { 2 - 3 } & \multicolumn{2}{c}{ Attended } & & \multicolumn{2}{c}{ Unattended } \\
\cline { 2 - 3 } \cline { 5 - 6 } & $M$ & $S D$ & & $M$ & $S D$ \\
\hline Short ISI & & & & \\
Central & .81 & .21 & .70 & .24 \\
Peripheral & .41 & .16 & .33 & .16 \\
Long ISI & & & & \\
Central & .88 & .17 & .86 & .17 \\
Peripheral & .43 & .20 & .40 & .13 \\
\hline
\end{tabular}

Note-ISI, interstimulus interval.

the results reflect a fairly good localization performance with minimal attentional resources.

\section{EXPERIMENT 2}

In the second experiment, we employed a dual task to investigate whether limiting attentional resources allocated for visual localization would also reduce localization precision. Participants were presented simultaneously with a central three-letter array and a small target that appeared in one of four possible peripheral locations. In the single-task condition, participants ignored the central stimulus and only localized the peripheral target. In the dual-task condition, they first localized the target and then indicated whether the central array contained a prespecified colored letter. Since nontarget letters in the array could be of the prespecified target color or shape, the task required the correct integration of shape and color to avoid illusory conjunctions. It was therefore assumed that the letter-identification task would consume a great deal of attentional resources (Treisman $\&$ Schmidt, 1982), thus substantially reducing the attention available for visual localization. The present manipulation is generally similar to that used by Prinzmetal et al. (1998). However, unlike Prinzmetal et al., who reduced location uncertainty by presenting all stimuli along the same perimeter, we maximized location uncertainty by presenting the target in a random position within one of four diagonal regions, the diameter of each subtending $4^{\circ}$ of visual angle. This advantage is especially important here, since we wanted to obtain a faithful quantitative estimate of the spread of localization responses for minimally attended stimuli with maximal location uncertainty.

\section{Method}

Participants. The participants were 10 paid undergraduates from Tel Aviv University. All had normal or corrected-to-normal vision.

Stimuli and Procedure. The stimuli and procedure were identical to those of Experiment 1, except for the changes noted below. On each trial, the participants were presented simultaneously with a peripheral target and a central three-letter array. The peripheral target, a small open circle subtending $0.26^{\circ}$ in diameter, appeared in one of four possible corners of a central imaginary square, the side of which subtended $5.6^{\circ}$ of visual angle. In each of the four lo- 
cations, the target appeared in a random position within an area, the diameter of which subtended $4^{\circ}$ of visual angle. The three-letter array was centered inside a rectangular white outline subtending $1^{\circ}$ in height and $3^{\circ}$ in width. Each array contained three different uppercase letters - one red, one green, and one white. The letters were sampled from the entire alphabet, and the colors were randomly assigned to the three letters, each of which subtended a visual angle of $.4^{\circ}$ in height and $.3^{\circ}$ in width. The participants were run under two conditions. In the dual-task condition, they first localized the peripheral target by touching the screen. They then pressed one of two keys to indicate whether the array contained a prespecified colored letter. In the single-task condition, the participants ignored the central array and only localized the peripheral target. Each trial consisted of the following sequence of events. First, either a red, green, or white target letter was presented at the center for $2 \mathrm{sec}$. Then, following a 1-sec blank interval, the letter array and the peripheral target appeared for $170 \mathrm{msec}$. On half of the trials, the array contained the prespecified target, and on the other half the array did not contain the target, but one of the letters had the target shape and another had the target color. Thus, each identification response was based on the conjunction of shape and color.

\section{Results and Discussion}

Figure 1 presents the distribution of localization responses around the four diagonal locations. In order to concisely describe the magnitude and direction of dispersion, all stimulus positions for each of the four diagonal locations were placed on a single origin. The figure contains the entire set of data-namely, the position of each response relative to the corresponding stimulus position for each participant on every trial. An overall ANOVA indicated a greater dispersion around the stimuli in the dual-task condition than around those in the single-task condition $[F(1,9)=14.87, p=.003]$. This finding replicates the results obtained by Prinzmetal et al. (1998). Table 3 presents the mean absolute localization deviations, as well as their component deviations along the radial and tangential diagonal axes. Figure 1 and Table 3 suggest that, as in the first experiment, the distribution of localization responses is scattered to a greater extent along the radial than along the tangential axis. The analysis confirmed this observation for the stimuli in the single-task condition $[F(1,9)=17.15, p<.003]$, as well as for those in the dual-task condition $[F(1,9)=$ $7.02, p<.03]$. Hence, additional analyses were carried out to assess the effects of attention on localization separately along the two axes. Unlike in the first experiment, these analyses showed that attention reduced localization dispersion along both the radial axis $[F(1,9)=15.55$, $p<.003]$ and the tangential axis $[F(1,9)=8.66, p=$ $.016]$.

Since Experiment 2 eliminated peripheral cues and minimized shifts of attention toward the target, it appears that the elongated distribution of localization responses is not produced by attentional shifts, but rather reflects a structural characteristic of the attentional field. The orientation of this elongated localization distribution is determined by the position of the peripheral stimulus relative to the center; indeed, there is a greater locational uncertainty along the axis connecting fixation to stimulus location than along the axis that is perpendicular to it.
Of major importance to the theoretical discussion presented below is the localization performance under the dual-task condition. The present results show that even with minimally attended stimuli and maximal location uncertainty, localization performance was fairly accurate. Although attentional resources were engaged in a concurrent demanding task, with no precue directing attention to the target, localization responses were not simply scattered anywhere on the screen. Instead, the absence of attention slightly expanded the area spanned by the localization responses, producing a mean localization error of only $.62^{\circ}$, thus suggesting that coarse localization is possible without attention.

\section{GENERAL DISCUSSION}

The present results reinforce previous findings (Prinzmetal et al., 1998; Tsal \& Bareket, 1999; Tsal \& Meiran, 1993) that demonstrate consistent effects of attention in reducing the spread of localization responses around the stimulus location.

Both experiments also show a greater dispersion along the radial axis than along the tangential one. Hence, the asymmetrical localization spread is not unique to the horizontal dimension (Tsal \& Bareket, 1999), but instead characterizes peripheral stimuli in general. One may argue that this asymmetrical distribution results from the fact that participants code the four diagonal directions as imaginary anchor points, thus facilitating localization along the tangential axis. Note, however, that such a bias might facilitate localization if the target is always presented in the precise diagonal position or very closely nearby, or more generally, if the magnitude of mislocalization exceeds the range along which the stimulus could be presented. However, in both experiments, the stimulus could appear along a tangential axis of $4^{\circ}$ ( $2^{\circ}$ toward each side from the diagonal point), whereas the mean localization error along the tangential axis was less than half a degree of visual angle. Thus, relying on these diagonal anchor points would only bias localization toward the diagonal, thereby increasing the magnitude of deviations for most stimuli except for those falling on, or very near, the diagonal axis.

The present finding argues against previous conceptions that postulate a single representation of location as a unitary entity. It suggests, instead, that the representation of location is made up of two components jointly required to specify peripheral locations. One component involves the azimuth or radial angle of the peripheral location relative to the center, and the second specifies the distance of the peripheral location from the center. The present pattern of results suggests that the system is provided with fairly precise information concerning the former but imprecise information concerning the latter. It appears that the general direction of the stimulus is available with minimal attention and that further attentional resources are needed in order to place the stimulus at the appropriate distance along this direction. 


\section{DEG.}

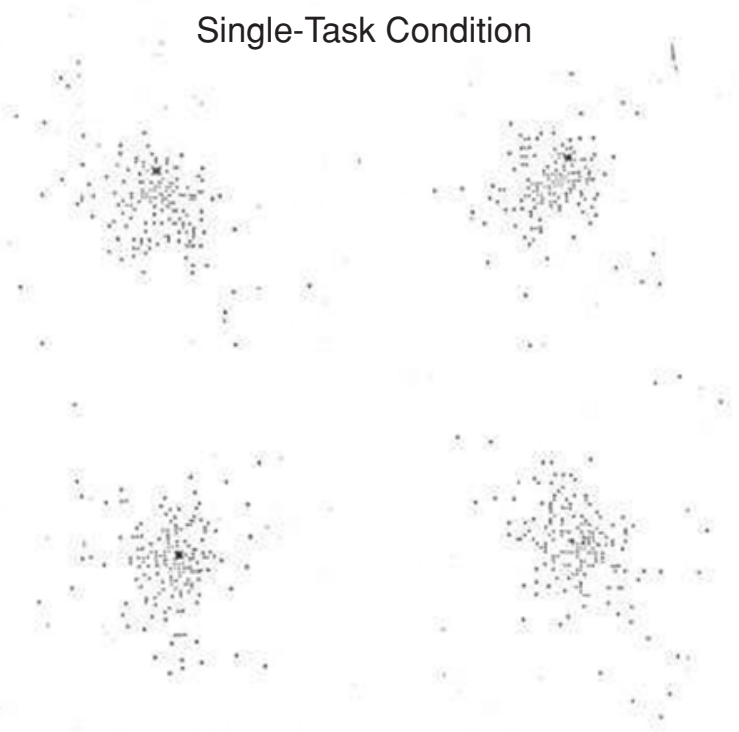

Dual-Task Condition

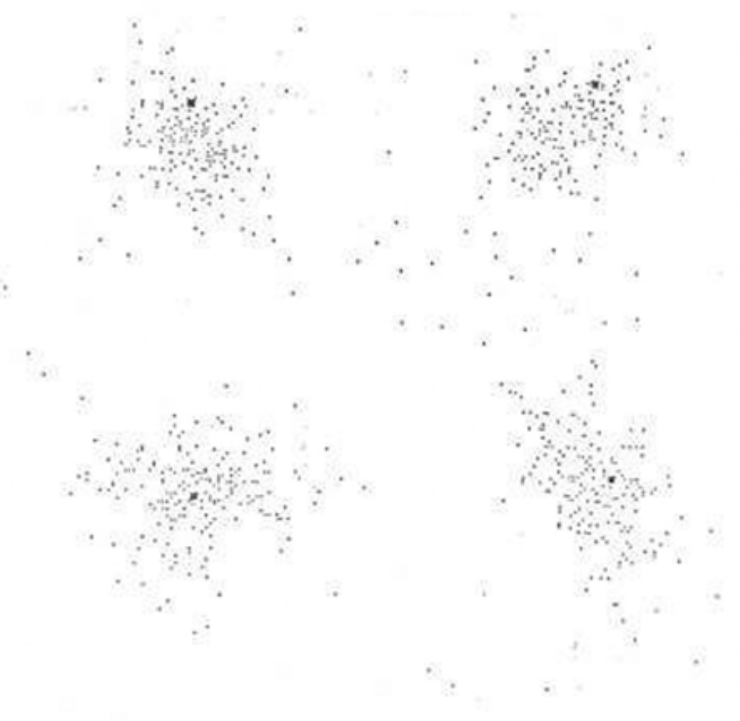

Figure 1. Distribution of localization responses around diagonal positions.

Most important, the present results indicate that coarse localization is possible with minimal attention. The assessment of localization accuracy depends on an arbitrary adjustment of a resolution criterion, where according to a very fine criterion, neither highly attended nor minimally attended stimuli were localized correctly, whereas according to a rough criterion, both were localized correctly. The pattern of localization responses clearly shows that the lack of attentional resources did not result in "incorrect" responses. That is, even with the maximally unattended stimuli in the dual-task condition of Experiment 2, when resources were engaged in a concurrent demanding task, with no precue directing attention to the target, localization responses were not simply scattered anywhere on the screen. Although the stimulus could appear unexpectedly in one of four remote peripheral locations, and in each within an area the diameter of which subtended $4^{\circ}$ of visual angle, the mean deviation 
Table 3

Experiment 2: Mean Localization Deviations in Degrees of Visual Angle

\begin{tabular}{llllll}
\hline & \multicolumn{2}{c}{$\begin{array}{c}\text { Single-Task } \\
\text { Condition }\end{array}$} & & \multicolumn{2}{c}{$\begin{array}{c}\text { Dual-Task } \\
\text { Condition }\end{array}$} \\
\cline { 2 - 3 } \cline { 5 - 6 } Deviation & $M$ & $S D$ & & $M$ & $S D$ \\
\hline Absolute & .46 & .16 & & .62 & .18 \\
Radial Axis & .33 & .11 & & .45 & .16 \\
Tangential Axis & .25 & .09 & .33 & .11 \\
\hline
\end{tabular}

in localizing the stimulus was $0.62^{\circ}$ of visual angle. Clearly, this pattern of responses is hardly the manifestation of a random distribution of localization responses, but rather reflects the capacity of the system to enable coarse localization with minimal attention.

Hence, the present finding supports an intermediate view that proposes that coarse localization is possible at the preattentive stage, whereas fine localization requires attention (Cohen \& Ivry, 1989, 1991; Newby \& Rock, 2001; Tsal et al., 1995; Tsal \& Shalev, 1996). The two present experiments clearly entailed different levels of attention. In the first experiment, in the highattention condition attention was maximally focused on the target location, and in the low-attention condition attention was distributed over a large area. In the second experiment, in the high-attention condition attention was distributed over a large area (similar to the low-attention condition of Experiment 1), and in the low-attention condition attention was distributed and, also, participants were engaged in a simultaneous demanding task. The various differences between the two procedures do not allow for cross-experiment comparisons, but the fact that significant effects of attention on localization performance were obtained in both experiments suggests that the localization of stimuli receiving differential amounts of attention represents different levels on a scale of localization accuracy, in which the gradual increase of resources (from focused to distributed in Experiment 1 and from distributed to diverted in Experiment 2) produces an increasing localization precision.

Below, we speculate on this relationship between attentional resources and localization precision. This proposed theoretical account is only partially tested in the present study. It is presented as a general framework that takes into account the various constraints of the visual system and is based on various theoretical notions that are widely accepted in the literature.

We propose that the distribution of responses to minimally attended stimuli reflects the operation of attentional receptive fields (ARFs) within which there is minimal resolution or spatial differentiation (Shalev \& Tsal, 2002; Tsal \& Shalev, 1996). The ARF provides only rough location information, and increasing attentional resources facilitates computations that gradually increase localization precision. What could be the theoretical basis for these hypothesized ARFs? Clearly, there is no immediate inherent relationship between attention and localization scale. Therefore, a proposed theoretical ex- planation that would go beyond just the common statement that attention improves a given perceptual operation, such as localization, at present requires speculation about how the structure of the visual system enables coarse localization without attention, and how attentional resources facilitate computations that produce fine localization.

The proposed mechanism should take into account the unavoidable contrast between the richness and diversity of the visual environment and the limitations imposed by the information processing system. One possible solution to this discrepancy entails a system that is capable of some form of low-level analysis carried out in parallel over the entire visual field, followed by the operation of a high-level analysis performed on the same input over a fairly small prespecified region in the field. We propose that the visual system is equipped with overlapping localization detectors that provide only a rough representation of location. This proposition is based on the evidence that central neurons involved in object localization have receptive fields that are substantially large, compared with the sensory resolution observed in behavioral experiments (e.g., Eurich \& Schwegler, 1997; Westheimer \& McKee, 1977).

Finer spatial discriminations of visual stimuli are achieved by integrating the outputs of adjacent detectors and computing their relative activation (Tsal et al., 1995). This notion of coarse coding is widely accepted in the literature (e.g., Ballard 1986; Eurich \& Schwegler, 1997; Hinton, McClelland, \& Rumelhart, 1986). According to this view, receptive fields at each location are highly overlapping and precise localization derives from decoding the population activity of the units with overlapping receptive fields (e.g., Eurich \& Schwegler, 1997; Fahle \& Poggio, 1981).

Figure 2 illustrates the operation of this system with various levels of attention. The figure presents the coarse detectors, each responding with variable degrees of activation to any of several possible stimuli, which slightly vary in location. The area that can activate a given detector defines the attentional receptive field of this detector. Stimuli appearing within that ARF can activate the detector with variable degrees of intensity. Any given stimulus is most likely to activate several overlapping detectors differentially. For example, the small black disk presented in the figure produces a minimal activation of Detector 1, an intermediate activation of Detector 2, and a higher activation of Detector 3 . This proposed localization system can produce sufficient output for the specification of the precise stimulus location. However, the assessment of the precise activation level of each detector requires attentional resources.

The notion that attention modulates local competition in the visual cortex has been proposed independently on the basis of theoretical (Niebur \& Koch, 1994; Tsotsos et al., 1995) and single-neuron studies (Desimone, 1998). It has been further proposed that attention modulates interactions between neurons with overlapping receptive fields (Moran \& Desimone, 1985). Only the very coarse 


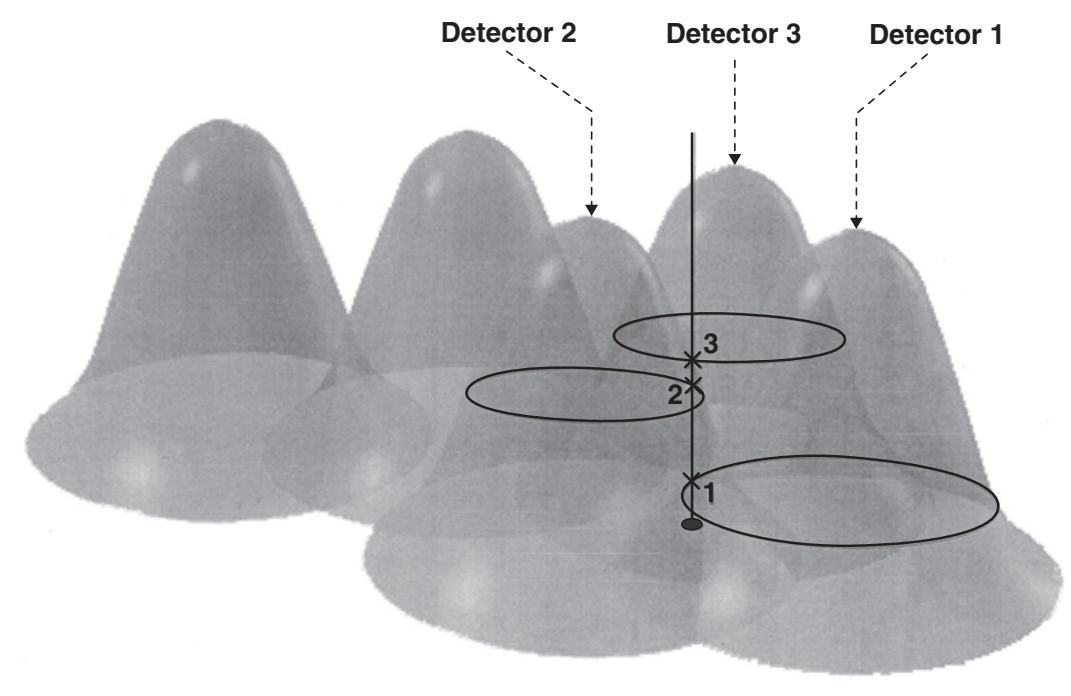

Figure 2. Attentional receptive fields mediated by coarse localization detectors. Preattentive localization: most highly activated detector. Attentive localization: relative activation of overlapping detectors.

activation level of each detector registers with minimal attention. Most often, this coarse activation enables only the assessment of the most highly activated detector. Thus, for example, when the stimulus is minimally attended, such as when attention is focused on a remote area in the visual field, the system automatically responds by signaling the operation of the most highly activated detector-namely, Detector 3. The system will consequently recognize that the stimulus can occur in any location falling within the region that can activate Detector 3-namely, ARF 3, thus producing a rough representation of location within the boundaries of this region. This system produces a fairly reliable but crude representation of the visual world that is not suitable for fine spatial discriminations. Thus, a minimally attended object may have lost its exact location coordinates, but one seems to be aware of roughly where it is.

This proposition is similar to the one made by Yuille and Grzywacz (1989), who suggested that fine localization may require attentional capacity, and without focused attention, the readout of the population activity may be simpler, akin to a winner-takes-all mechanism (e.g., Lee, Itti, Koch, \& Braun, 1999). According to this view, localization is determined primarily by the most active unit rather than by the full information contained by all the overlapping units, and consequently localization precision is diminished. When limited resources are allocated to the stimulus location, such as when attention is distributed over an area encompassing this location, a moderately coarse evaluation of the activation levels is possible. A computation of the relative activation of the various stimulated detectors would define a small localization area. Since the activation levels of the detectors are not yet precisely assessed, this computation would not specify a precise localization value, but rather a small range of localization values. As attention narrows on the target and more resources are allocated to its location, a finer evaluation of the activation levels becomes possible and the range of localization values becomes smaller, thus resulting in finer localization. Finally, when maximal resources are available, such as when attention is focused on the stimulus, its location can be precisely determined. For example, in Figure 2, the system can compute the precise relative activation of Detectors 1,2, and 3 which exactly specifies the location of the small black disk.

The model presented above has several advantages. First, it explains two major findings obtained in the present study: the efficient coarse localization under minimal attention conditions and the gradual improvement in localization with increasing attentional resources. Second, it integrates various significant propositions made in the behavioral, neurophysiological, and computational literatures. Third, it offers a parsimonious account for both attended and unattended localization by postulating a single mechanism that integrates parallel low-level analysis with high-level analysis performed on the same input over a small region in the field.

\section{REFERENCES}

BALlard, D. H. (1986). Cortical connections and parallel processing: Structure and function. Behavioral \& Brain Sciences, 9, 67-120.

CoHEN, A., \& IVRY, R. (1989). Illusory conjunctions inside and outside the focus of attention. Journal of Experimental Psychology: Human Perception \& Performance, 15, 650-663.

CoHen, A., \& Ivry, R. (1991). Density effects in conjunction search: Evidence for a coarse location mechanism of feature integration. Journal of Experimental Psychology: Human Perception \& Performance, 17, 891-901. 
Desimone, R. (1998). Visual attention mediated by biased competition in extrastriate visual cortex. Philosophical Transactions of the Royal Society of London: Series B, 353, 1245-1255.

Eurich, C. W., \& Schwegler, H. (1997). Coarse coding: Calculation of the resolution achieved by a population of large receptive field neurons. Biological Cybernetics, 76, 357-363.

FAHLE, M., \& Poggio, T. (1981). Visual hyperacuity: Spatiotemporal interpolation in human vision. Philosophical Transactions of the Royal Society of London: Series B, 213, 451-477.

Hinton, G. E., McClelland, J. L., \& Rumelhart, D. E. (1986). Distributed representations. In D. E. Rumelhart, J. L. McClelland, \& the PDP Research Group (Eds.), Parallel distributed processing: Explorations in the microstructure of cognition. Vol. 1: Foundations (pp. 77109). Cambridge, MA: MIT Press.

JONIDES, J. (1981). Voluntary vs. automatic control over the mind's eye's movement. In J. B. Long \& A. D. Baddeley (Eds.), Attention and performance IX (pp. 187-204). Hillsdale, NJ: Erlbaum.

LeE, D. K., ItTi, L., Koch, C., \& Braun, J. (1999). Attention activates winner-takes-all competition among visual filters. Nature Neuroscience, 2, 375-381.

Moran, J., \& Desimone, R. (1985). Selective attention gates visual processing in the extrastriate cortex. Science, 229, 782-784.

Newby, E. A., \& Rock, I. (2001). Location and attention. Quarterly Journal of Experimental Psychology, 54A, 155-168.

Niebur, E., \& Koch, C. A. (1994). A model for the neuronal implementation of selective visual attention based on temporal correlation among neurons. Journal of Computational Neuroscience, 1, B141-B158.

Prinzmetal, W., Amiri, H., Allen, K., \& Edwards, T. (1998). Phenomenology of attention: 1. Color, location, orientation, and spatial frequency. Journal of Experimental Psychology: Human Perception \& Performance, 24, 261-282.

SAGi, D., \& Julesz, B. (1985). "Where" and "what" in vision. Science, 228, 1217-1219.
Shalev, L., \& Tsal, Y. (2002). Detecting gaps with and without attention: Further evidence for attentional receptive fields. European Journal of Cognitive Psychology, 14, 3-26.

Treisman, A., \& Gormican, S. (1988). Feature analysis in early vision: Evidence from search asymmetries. Psychological Review, 95, 15-48.

Treisman, A., \& SchmidT, H. (1982). Illusory conjunctions in the perception of objects. Cognitive Psychology, 14, 107-141.

TsAL, Y. (1989). Do illusory conjunctions support the feature integration theory? A critical review of theory and findings. Journal of Experimental Psychology: Human Perception \& Performance, 15, 394 400.

TsAL, Y., \& BAREKET, T. (1999). Effects of attention on localization of stimuli in the visual field. Psychonomic Bulletin \& Review, 6, 292296.

Tsal, Y., \& MeIran, N. (1993, November). Toward a resolution theory of visual attention. Paper presented at the 34th Annual Meeting of the Psychonomic Society, Washington, DC.

Tsal, Y., Meiran, N., \& LAmy, D. (1995). Toward a resolution theory of visual attention. Visual Cognition, 2, 313-330.

TSAL, Y., \& SHALEV, L. (1996). Inattention magnifies perceived length: The attentional receptive field hypothesis. Journal of Experimental Psychology: Human Perception \& Performance, 22, 233-243.

Tsotsos, J. K., Culhane, S. M., Wai, W. Y. K., LaI, Y., Davis, N., \& Nuflo, F. (1995). Modeling visual attention via selective tuning. Artificial Intelligence, 78, 507-547.

Westheimer, G., \& McKeE, S. P. (1977). Spatial configurations for visual hyperacuity. Vision Research, 17, 941-948.

Yuille, A. L., \& GRZYWACZ, N. M. (1989). A winner-take-all mechanism based on presynaptic inhibition feedback. Neural Computation, 1, 334-347.

(Manuscript received August 7, 2003; revision accepted for publication June 18, 2004.) 